\title{
Increasing incidence of pertussis in Brazil: a retrospective study using surveillance data
}

\author{
Lucas Melo Guimarães ${ }^{1,2}$, Eduilson Lívio Neves da Costa Carneiro ${ }^{3}$ and Filipe Anibal Carvalho-Costa ${ }^{1,2^{*}}$
}

\begin{abstract}
Background: Many countries have reported an increase in the incidence of pertussis, which has become a global public health concern.

Methods: In this study, the epidemiology of pertussis in Brazil was assessed retrospectively using surveillance data gathered from case notification forms from 2007 to 2014.

Results: From 2007 to 2014, 80,068 suspected cases of pertussis were reported in Brazil. Of these, 24,612 (32\%) were confirmed by various criteria. The annual distribution of confirmed cases demonstrated a significant increase in incidence rate since 2012. A seasonal pattern in which cases occur most frequently between the end of spring and midsummer has been identified. Among the confirmed cases, $34.5 \%$ occurred in infants aged 0-2 months, $22.4 \%$ occurred in infants aged 3-6 months, $21 \%$ occurred in children aged 7 months to 4 years, and $8 \%$ were reported in adults $>21$ years. Of the confirmed cases, $47.2 \%$ met only clinical criteria, $15.5 \%$ met clinical and epidemiological criteria, and $36.6 \%$ were confirmed in a laboratory. The overall case fatality rate was $2.1 \%$, reaching $4.7 \%$ among infants aged $0-2$ months. The complications most commonly reported in the notification forms were pneumonia, encephalitis, dehydration, otitis, and malnutrition. Of the confirmed cases, $23.1 \%$ occurred in subjects who received at least 3 doses of the pertussis vaccine. Within this group, there were 1098 infants aged 7 to 15 months and 2079 children aged 16 months to 4 years. In 2012, 18 states did not achieve $95 \%$ immunization coverage, a number that dropped to 10 and 6 in 2013 and 2014, respectively.
\end{abstract}

Conclusions: Brazil's main challenges in facing pertussis resurgence will be to offer the best quality medical attention to reduce mortality, to improve the infrastructure for laboratory diagnosis and to increase vaccination coverage. Additional studies to assess the effectiveness of the current vaccination schedule and basic research on the genetics and evolution of circulating B. pertussis strains are also needed.

Keywords: Pertussis, Bordetella pertussis, Epidemiology, Vaccination, Brazil

\section{Background}

An increasing incidence of pertussis (whooping cough) has been reported in many countries and currently represents a global public health concern $[1,2]$. The disease is a potentially lethal, highly contagious respiratory tract infection caused by the gram-negative bacteria Bordetella pertussis. B. bronchiseptica, B. parapertussis, and B. holmesii can also cause the disease in humans [3, 4]. A pertussis-like syndrome can be caused by respiratory viruses, mycoplasmas,

\footnotetext{
*Correspondence: guaratiba@ioc.fiocruz.br

'Regional Office Oswaldo Cruz Foundation (Fiocruz), Teresina, Piauí, Brazil ${ }^{2}$ Laboratory of Epidemiology and Molecular Systematics, Oswaldo Cruz Institute, Fiocruz, Rio de Janeiro, Brazil

Full list of author information is available at the end of the article
}

and other bacteria, such as Haemophilus influenzae and Streptococcus pneumoniae [5].

Pertussis has an incubation period ranging from 5 to 21 days. $B$. pertussis infects the respiratory epithelium of trachea, bronchi and bronchioles, causing an accumulation of mucus and debris in the airways [6]. Clinically, the disease is characterized by a paroxysmal cough and fever and is frequently complicated by episodes of apnoea and cyanosis [7]. The majority of fatal cases have are among patients who develop bronchopneumonia caused by B. pertussis or a co-infection with other bacteria [6].

Pertussis is a vaccine-preventable disease. The incidence has been reduced drastically in all countries that have achieved satisfactory levels of vaccine coverage. Pertussis 
vaccines are combined with diphtheria and tetanus toxoids to produce the diphtheria-tetanus-pertussis (DTP) trivalent vaccine. More recently, adding the hepatitis $\mathrm{B}$ virus, $H$. influenzae type B antigens, and/or inactivated poliovirus has led to the development of tetravalent and pentavalent vaccines [8].

The first generation of vaccines, still widely used in developing countries, contains heat-killed whole $B$. pertussis cells $(\mathrm{wP})$ obtained from cultures. These vaccines are highly immunogenic and effective, although they are associated with potentially severe side effects such as febrile seizures [9]. Acellular (aP) vaccines are used in developed countries and are significantly less reactogenic, containing distinct combinations of three to five of the following antigens: pertussis toxin, filamentous hemagglutinin, pertactin, fimbrial antigen 2, and fimbrial antigen 3 [10-12].

In 2002, the Brazilian National Immunization Program (NIP) replaced the first three doses of the diphtheria-tetanus-whole pertussis (DTwP) vaccine with the tetravalent vaccine $\mathrm{DTwP}+H$. influenzae type $\mathrm{B}$ (DTwP-Hib). In 2012, this vaccine was replaced by the pentavalent vaccine $\mathrm{DTwP}+H$. influenzae type $\mathrm{B}+$ hepatitis B (DTwP-Hib-HBV). Currently in Brazil, pertussis immunization is with 3 doses of the DTwP-Hib$\mathrm{HBV}$ vaccine given at 2, 4 and 6 months of age, followed by two boosters with DTwP at 15 and 48 months of age. The diphtheria-tetanus-acellular pertussis (DTaP) is given only to infants who have had severe reactions to the DTwP or in private vaccine clinics, and 2014, the NIP began providing it to pregnant women.

Several hypotheses have been proposed to explain the resurgence of the disease, including a decrease in the immunogenicity of aP vaccines, which would lead to early weaning of immunity and result in B. pertussis circulating among adolescents and adults and spreading from them to young infants who are not fully immunized. It has also been proposed that $B$. pertussis may have undergone a genetic evolution associated with the selective pressure of vaccines. A significant increase in the pertussis incidence rate has been observed in Brazil in recent years [13]. This study aims to describe aspects of the pertussis resurgence in Brazil, including the spatiotemporal distribution, case fatality rates, confirmation criteria and patient vaccination status.

\section{Methods}

The epidemiology of pertussis in Brazil was studied using surveillance data that were retrospectively gathered from case notification forms from 2007 to 2014. Data that are generally not freely accessible were made available for this study by the Brazilian Ministry of Health. Brazil has a population of 202,768,562 inhabitants spread over $8,515,767 \mathrm{~km}^{2}$ and is divided into five geographical regions: the North (which corresponds to the Amazon region with 16,983,485 inhabitants), the Northeast $(53,081,510$ inhabitants), the Midwest (14,993,194 inhabitants), the Southeast (84,465,579 inhabitants) and the South $(27,465,289$ inhabitants). Brazil has 26 federative units (states) and one Federal District.

Information concerning the vaccination coverage rates were collected from the website of the Department of Informatics of the Unified Health System (Datasus) [14]. Datasus calculates the immunization coverage rates as the number of children with complete basic scheme in the target age for a particular type of vaccine/number of children in the target age X 100 .

Pertussis is a reportable disease in Brazil. All suspected cases treated at the Unified Health System are reported using standardized forms sent to the Information System for Notifiable Diseases (SINAN) of the Ministry of Health. Pertussis notification forms have many variables, including demographic and clinical data, treatment, confirmation criteria, control measures adopted, pertussis vaccination status, hospital discharge and death. This study was conducted with the data available in pertussis reporting forms sent to the Ministry of Health of Brazil from 2007 to 2014.

In Brazil, suspected pertussis cases are confirmed or discarded at the health unit on the basis of clinical, epidemiological or laboratory criteria. A case is confirmed with clinical criteria if the blood count presents 20,000 or more leukocytes $/ \mu \mathrm{L}$ and 10,000 or more lymphocytes $/ \mu \mathrm{L}$, with a negative or absent culture, lack of epidemiological link, or without confirmation of aetiology. Epidemiological criteria are met with a confirmed case of pertussis via laboratory testing, which is performed between the beginnings of the catarrhal period until three weeks after the beginning of the disease paroxysmal period. Laboratory criteria are based on the isolation of $B$. pertussis using a culture of nasopharyngeal secretion or positive polymerase chain reaction (PCR). In a variable proportion of patients, a sample of nasopharyngeal secretion is collected and then forwarded to the Central Laboratories of Public Health (LACENs). Confirmation rates were calculated as the number of confirmed cases (by any criteria)/number of notified (suspected) cases.

Population data from the Brazilian Institute of Geography and Statistics, available at www.ibge.gov.br, were used for calculation of incidence rates as the number of pertussis confirmed (by any criteria) cases/population in a defined municipality-state-whole country in a specific year $\mathrm{X}$ 100,000 inhabitants. The spatio-temporal and age distribution of cases was plotted and maps were produced with Quantum Geographic Information System (QGIS) software (QGIS Development Team, available at http://www.qgis.org/en/site/). Case fatality rates in different regions, years, and age groups were calculated as the number of deaths/ number of confirmed cases X 100. Complications that led to fatalities were assessed. We also examined the vaccination status of notified cases, observing the utilization of DTwP-Hib (tetravalent) vaccine until May 2012, followed 
by the implementation of DTwP-Hib-HBV (pentavalent) vaccine in June 2012. Children were considered vaccinated if they had received three or more doses of DTwP-Hib or DTwP-Hib-HBV. The proportion of vaccinated subjects among confirmed and discarded cases was compared through the chi-square test with a statistical significance of $5 \%$. Analyses were performed with EpiInfo 7.1.2.0 (Centers for Disease Control and Prevention, Atlanta, Georgia, USA).

Descriptive statistics are presented, with the distribution of cases by month, year and state, as well as the frequencies of distinct diagnostic criteria used to confirm the cases. The mean incidence rates of pertussis were calculated and presented. The frequencies of clinical complications in the cured group and in the group that evolved to death were compared with chi-square test. The confirmation rates in vaccinated and unvaccinated groups in distinct age strata were also compared using the chi-square test. Statistical significance was established at $p<0.05$.

The study was approved by the Research Ethics Committee of the Oswaldo Cruz Institute/Fiocruz, license number 39406914.0.0000.5248.

\section{Results}

\section{Confirmed cases, incidence rates, spatio-temporal}

\section{and age distribution of pertussis in Brazil}

Between January 2007 and December 2014, 80,068 suspected cases of pertussis were reported in Brazil. Of these, 24,612 (32\%) were confirmed using clinical, epidemiological or laboratory criteria. The annual distribution of confirmed cases demonstrated a significant increase in the number of cases from 2012. The yearly average of confirmed cases was 1226 per year between 2007 and 2011, reaching 6161 per year between 2012 and 2014. The confirmation rate was not related to the increase in the number of cases being registered; the highest rate of confirmation was in 2009 (34.7\%), and the lowest was in 2010 (29.6\%). The annual distribution of reported, and the confirmed cases and confirmation rates are depicted in Fig. 1. The distribution of cases by month showed an increase in the period from November to February, as shown in Fig. 2. Therefore, a seasonal pattern, in which cases occur most frequently between the end of spring and midsummer in Southern Hemisphere, has been identified. This seasonality was observed in all five Brazilian regions, but seems to be more marked in the South and Southeast. The annual incidence rates between 2007 and 2010 ranged from 0.32 cases per 100,000 to 0.75 per 100,000, showing an increase in 2011 and reaching 1.17 per 100,000 , and then rising to 2.81 per 100,000 in $2012,3.2$ per 100,000 in 2013 , and 3.25 per 100,000 in 2014 (not shown). During the epidemic period, the mean annual incidence rate was 2.6 cases per 100,000 , compared with 0.51 per 100,000 in the period from 2007 through 2010.

With respect to the distribution of cases by state, the states of São Paulo, Espírito Santo, Rio Grande do Sul and Paraná accounted for $53.7 \%(n=13,216)$ of the confirmed cases of pertussis. During the epidemic period, when there were 19,522 confirmed cases, the Southeast region recorded $47.7 \%(n=9311)$, followed by the South with $20.5 \%(n=4000)$ and the Northeast with $20.4 \%(n=3988)$ (data not shown). The annual evolution of pertussis incidence rates from 2007 to 2014 in the different Brazilian municipalities is shown in the maps in Fig. 3. An increased incidence in many municipalities can be observed in 2012 .

By studying the distribution of cases by age, we aimed to identify the proportion of cases in age groups in which immunization from vaccines is not expected. These groups included infants aged 0 to 2 months and

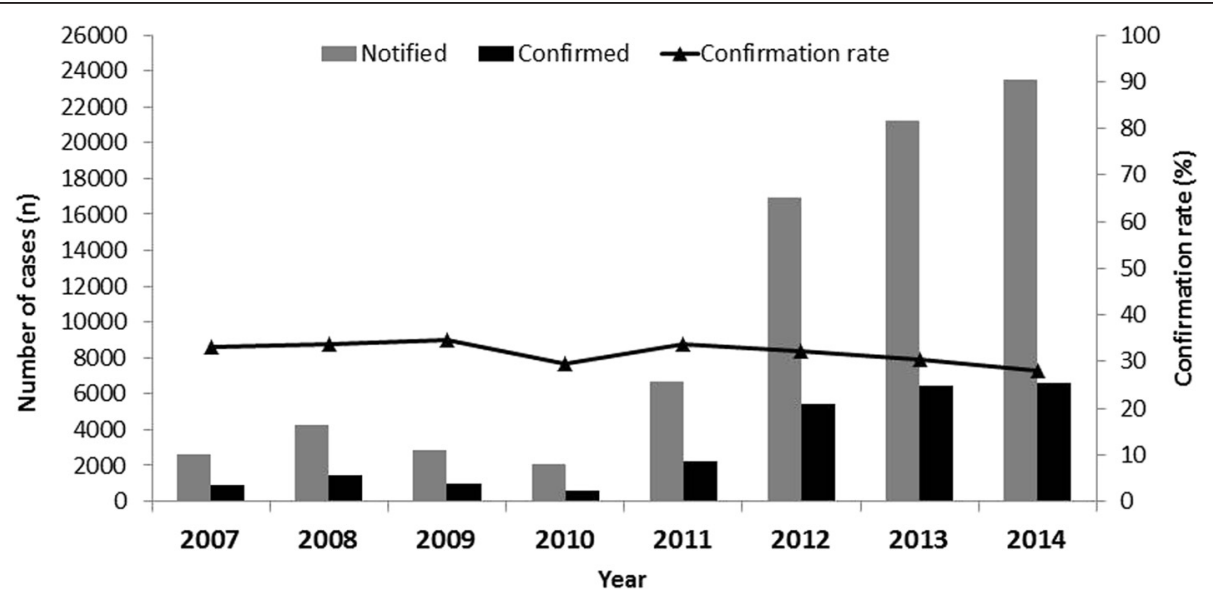

Fig. 1 Absolute numbers of reported and confirmed cases of pertussis and confirmation rates (confirmed cases/reported cases X 100) in Brazil, from 2007 to 2014 per year 


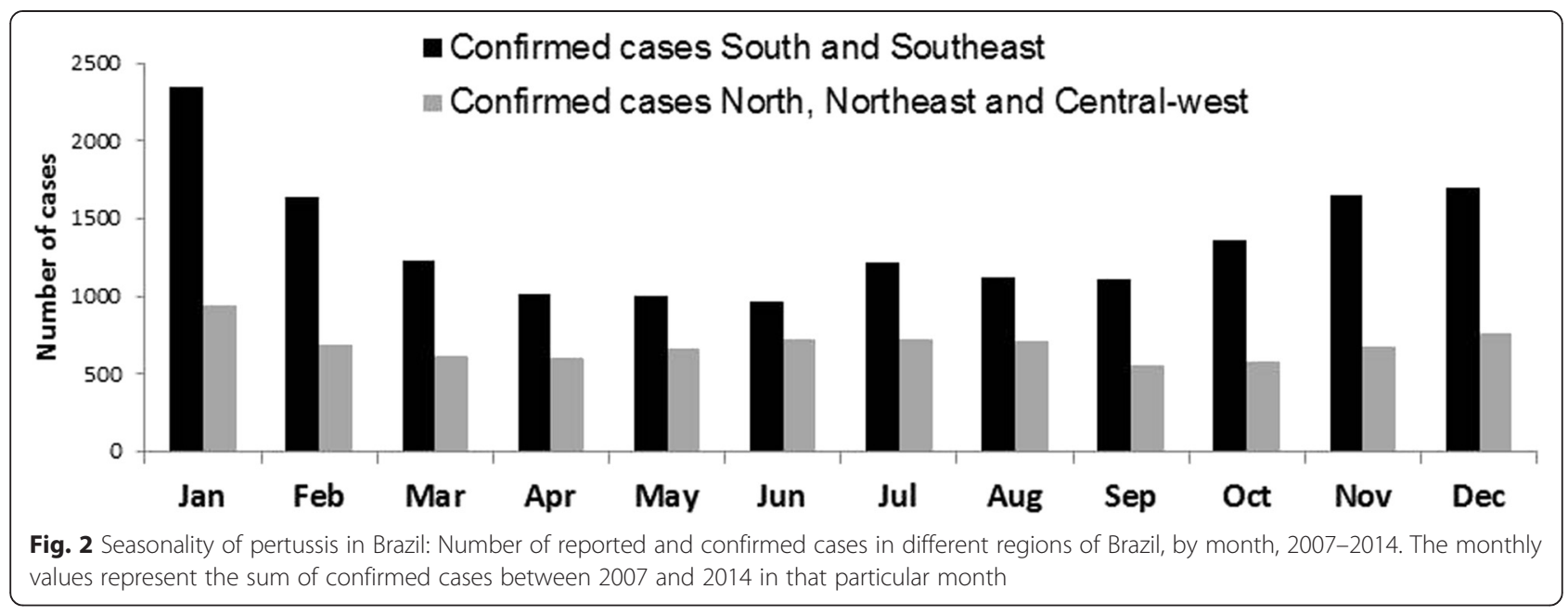

adults over the age of 21 years. The distribution is shown in distinct years in Fig. 4. Among the confirmed cases, $34.5 \%(n=8491)$ occurred in infants aged 0 to 2 months, and approximately $8 \%(n=1969)$ were reported in adults older than 21 years of age. In observing other age groups, it was noted that $22.4 \%(n=5513)$ were children aged 3 to 6 months, i.e., in the process of immunization, while $21 \%(n=5168)$ occurred in children aged 7 months to 4 years old, who are expected to be immunized. It is observed that in the period 2012-2014, the proportion of cases in children aged 0 to 6 months decreased, while the proportion of the reported pertussis cases among children aged 16 months to 4 years and 5 to 14 years increased. Among the confirmed cases, 55.4\%

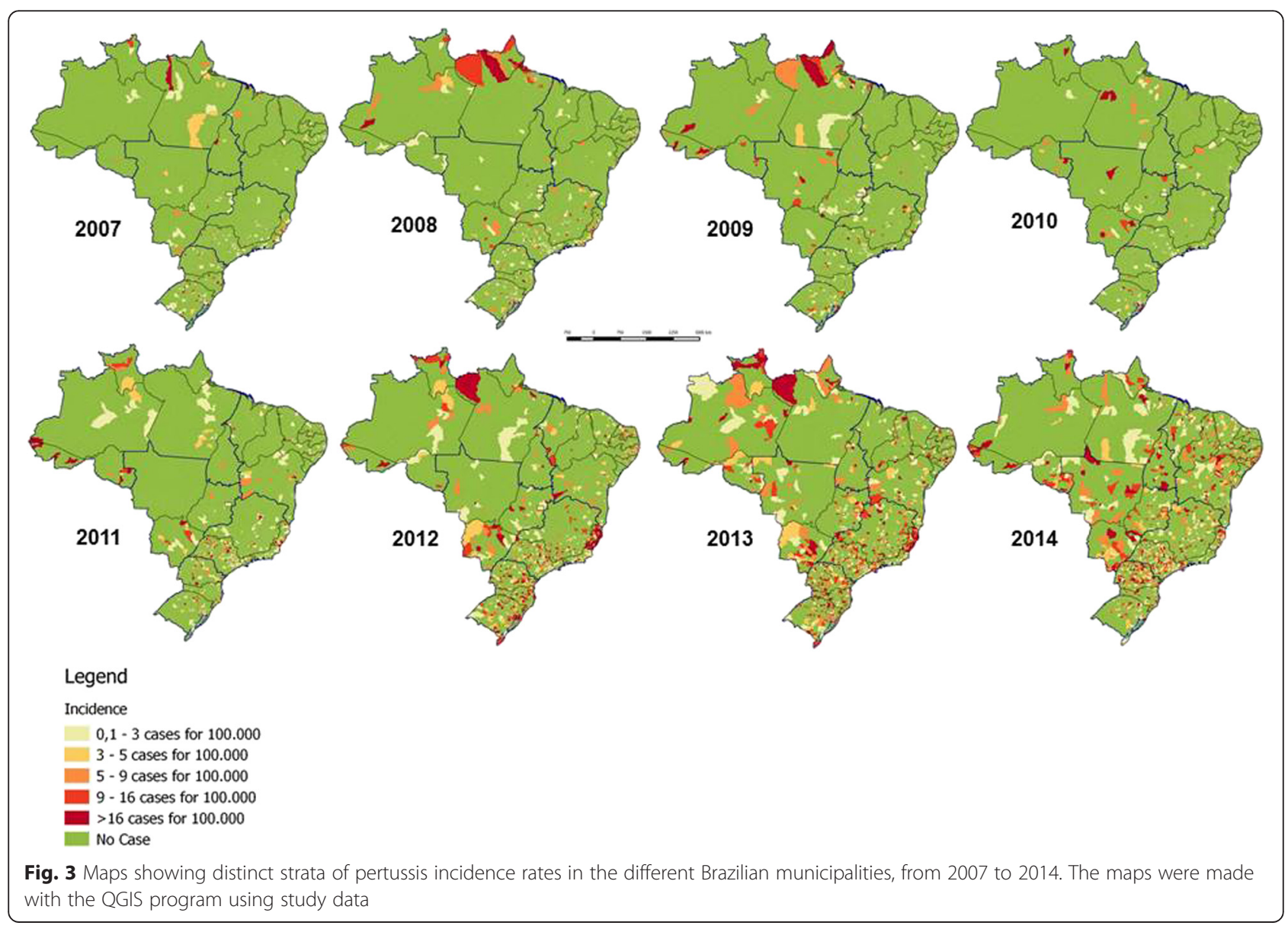




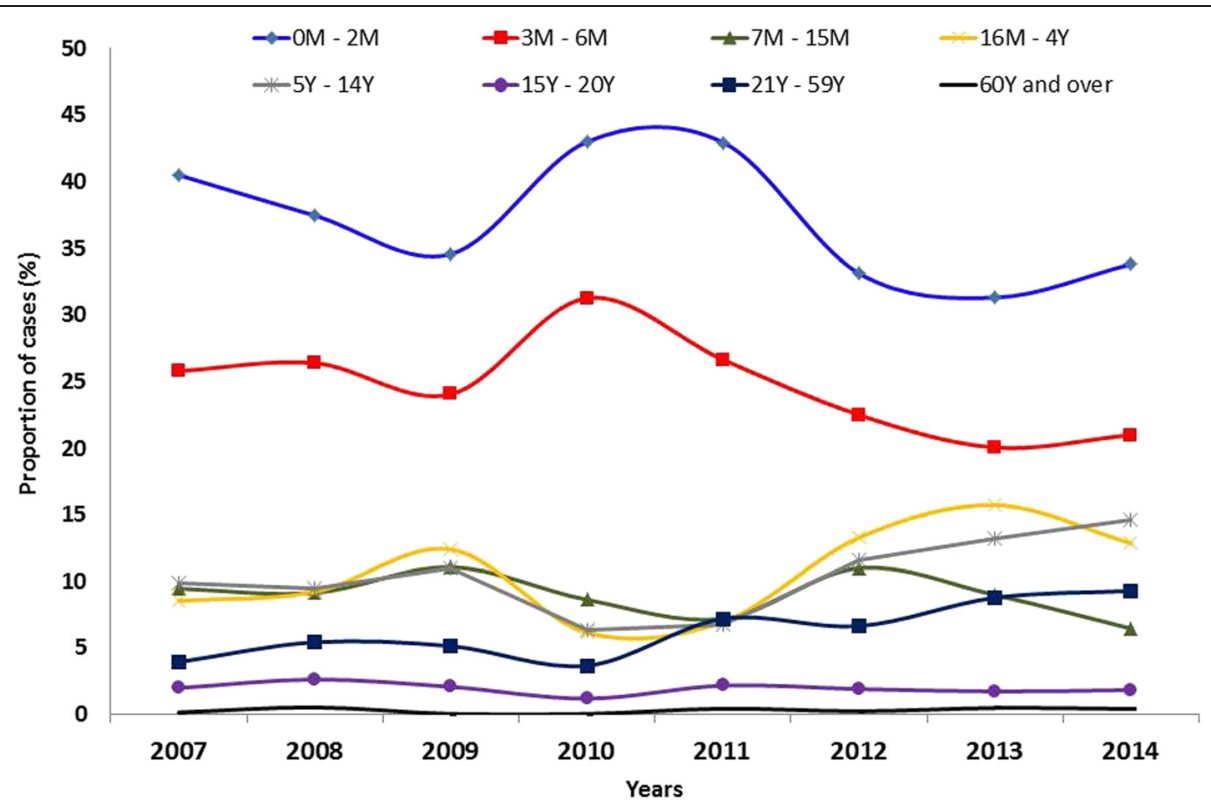

Fig. 4 Distribution of confirmed cases of pertussis by age group from 2007 to 2014. The values represent the proportion of cases registered in each age group per year

$(n=13,626)$ occurred in females, $0.5 \%(n=73)$ of whom were pregnant.

\section{Confirmation criteria for pertussis cases in Brazil}

It was observed that $47.2 \%(n=11,606)$ of the confirmed cases met only the clinical criteria, $15.5 \%(n=3821)$ met clinical and epidemiological criteria, and $36.6 \%(n=9017)$ were laboratory confirmed by various methodologies, most frequently the culture for $B$. pertussis of nasopharyngeal secretions. Among the confirmed cases, the laboratory confirmation rate ranged from 24.9 \% (2009) to $49.5 \%$ (2011). Important regional differences were also observed; São Paulo showed the highest rates, reaching $80.2 \%$ of laboratory confirmations over the period (Fig. 5).

\section{Complications and case fatality rates}

The overall case fatality rate among confirmed cases was $2.1 \%$ (528 per 24,612$)$. However, it was observed

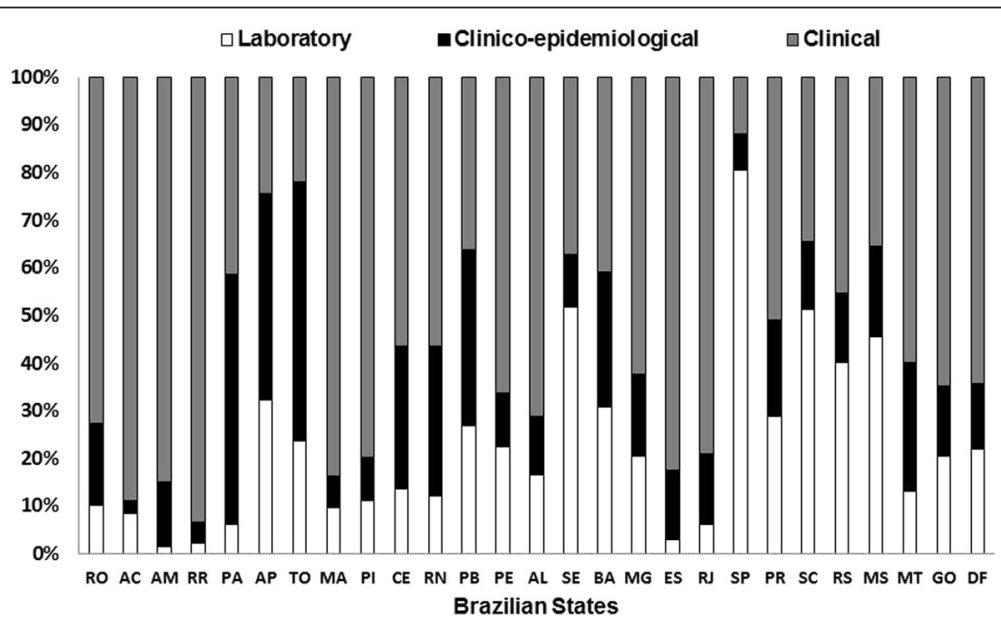

Fig. 5 Distribution of the criteria used to confirm the diagnosis of pertussis in Brazil, 2007-2014. The values represent the proportion of cases that were confirmed by each criteria, by state. $\mathrm{RO}=$ Roraima, $\mathrm{AC}=\mathrm{Acre}, \mathrm{AM}=$ Amazonas, $\mathrm{RR}=$ Roraima, $\mathrm{PA}=$ Pará, $\mathrm{AP}=\mathrm{Amapá}, \mathrm{TO}=\mathrm{Tocantins}, \mathrm{MA}=\mathrm{Maranh}$ ão, $\mathrm{PI}=$ Piauí, $\mathrm{CE}=$ Ceará, $\mathrm{RN}=$ Rio Grande do Norte, $\mathrm{PB}=$ Paraíba, $\mathrm{PE}=$ Pernambuco, $\mathrm{AL}=$ Alagoas, $\mathrm{SE}=$ Sergipe, $\mathrm{BA}=$ Bahia, $\mathrm{MG}=$ Minas Gerais, ES = Espírito Santo, RJ = Rio de Janeiro, SP = São Paulo, PR = Paraná, SC = Santa Catarina, RS = Rio Grande do Sul, MS = Mato Grosso do Sul, MT = Mato Grosso, $\mathrm{GO}=$ Goiás, DF = Distrito Federal (Federal District) 
that among children under 3 months of age, mortality was substantially higher, reaching $4.7 \%$ (401/8494). Figure 6 shows the annual distribution of pertussisassociated deaths, the evolution of case fatality rates from 2007 to 2014, and the mortality in specific age groups. A comparison of the case fatality rates in different regions of Brazil demonstrates the lowest rates in the South (1.3\%); Rio Grande do Sul state registered the lowest fatality rate $(0.8 \%)$. The Midwest region registered the highest case fatality rate among the macro-regions (2.5\%); however the state with the highest proportion of deaths was Roraima (North Region), which had a fatality rate of $11.4 \%$ (Fig. 7). The complications most commonly reported in the notification forms included pneumonia, encephalitis, dehydration, otitis, and malnutrition. In Table 1, we compare the frequency of these complications reported among the patients who progressed to hospital discharge or death in different age groups. Significantly higher frequencies of pneumonia, encephalitis, dehydration and malnutrition were observed in the group of children who progressed to death.

\section{Vaccination status of notified and confirmed pertussis cases and pertussis vaccine coverage}

It was noted that 10,600 confirmed cases of pertussis ( $43 \%$ of the total) occurred in people over the age of 7 months (the age group that should have received a minimum of 3 doses of vaccine against pertussis). Of this group, there was information about the vaccination status of 6948 subjects. These cases were divided into two groups: fully vaccinated (3 doses), with 5687 subjects, and incompletely vaccinated (less than 3 doses), with 1261 individuals. Thus, at least $23.1 \%$ of the confirmed pertussis cases occurred in subjects who had received at least 3 doses of the pertussis vaccine. Within this group, there were 2079 children aged 16 months to 4 years and 1098 infants aged 7-15 months. Thus, it can be considered that $12.9 \%$, i.e., $(2079+1098) / 24.612$ cases of pertussis confirmed in Brazil between 2007 and 2014, occurred in fully immunized infants and children aged 7 months to 4 years. Among these 3177 cases, 911 were confirmed using laboratory criteria; this value corresponds to $3.7 \%$ of confirmed pertussis cases in Brazil between 2007 and 2014. Among the 1261 cases reported

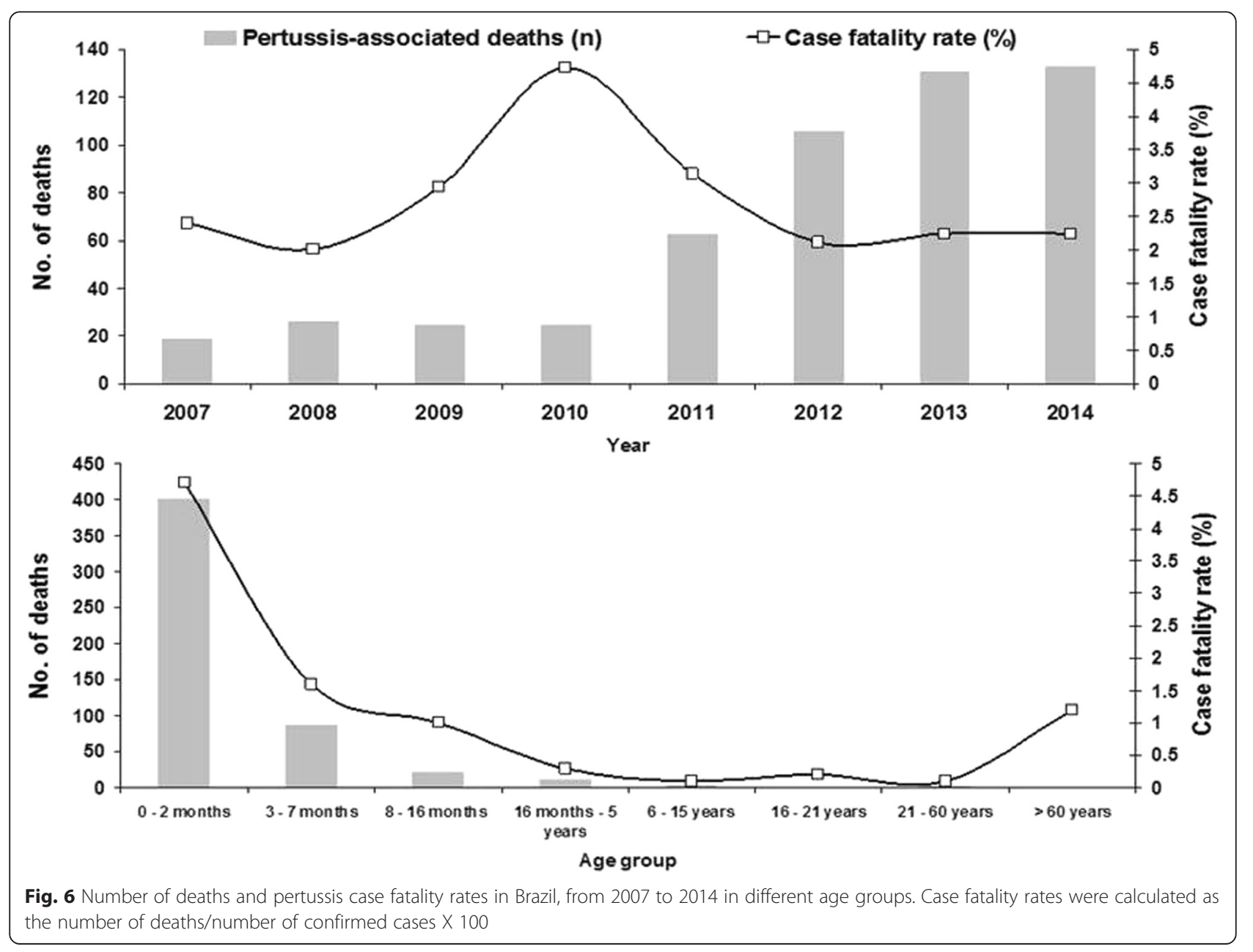




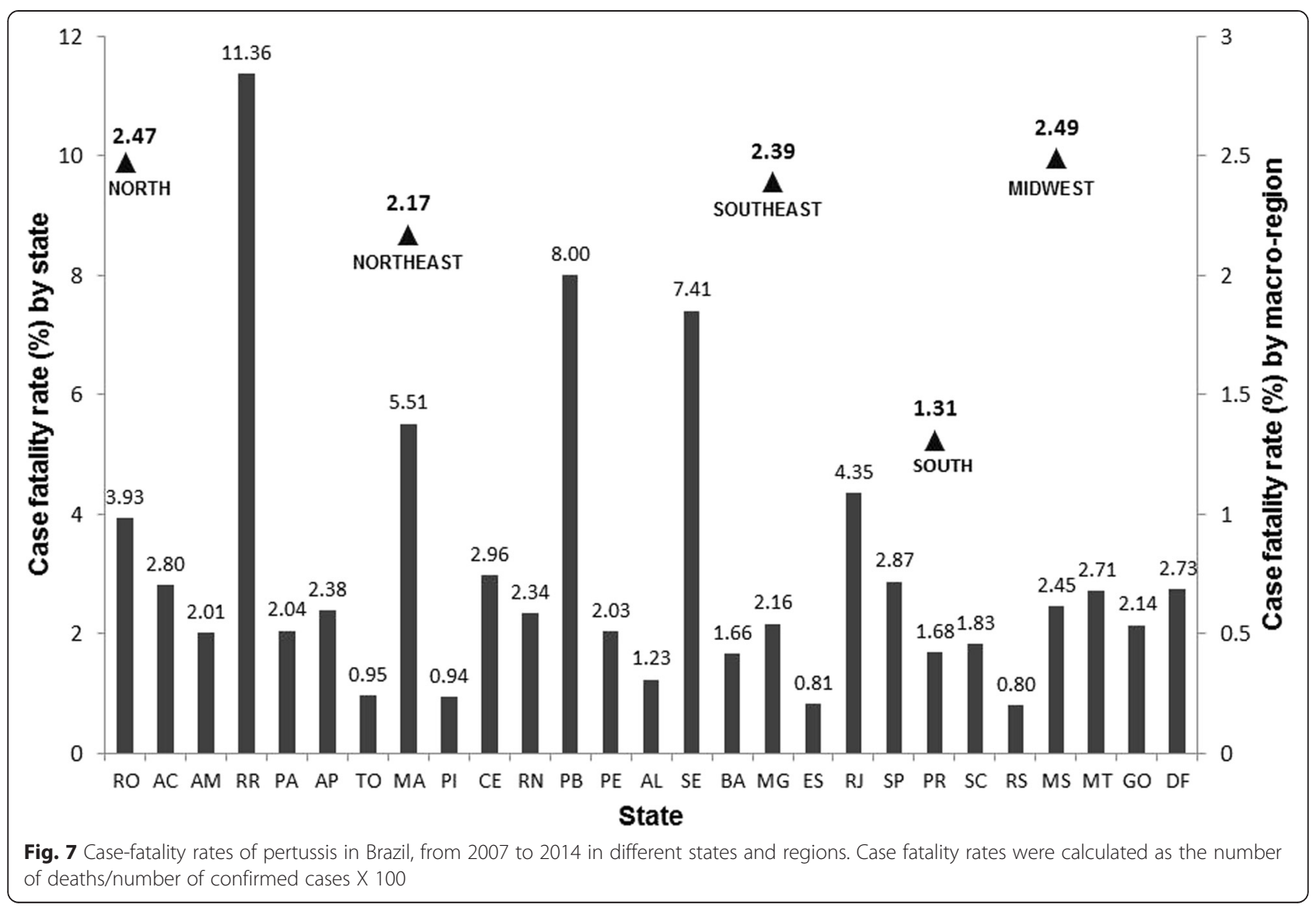

in incompletely vaccinated subjects older than 7 months of age, the predominant age group was between 7 and 15 months $(n=515)$.

Table 2 shows that the confirmation rates were significantly lower in children with a complete vaccination schedule (3 or more doses) compared with those with incomplete immunization (2 doses or less), both in the group aged 7 to 15 months and in the group aged 16 months to 4 years. However, beginning in June 2012, which coincided with the replacement of DTwP-Hib by the DTwP-Hib-HBV vaccine, the difference between the confirmation rates among completely and incompletely vaccinated children decreased, primarily in the group aged 16 months to 4 years.

In 2007, two states did not reach $95 \%$ vaccination coverage, and this number increased to six states in 2008. In 2012, 18 states did not achieve $95 \%$ immunization coverage, a number that dropped to ten and six in 2013 and 2014, respectively (Fig. 8). As presented in Fig. 9, during the pre-epidemic period (2007-2011), there was a trend of correlation between pertussis incidence and vaccine coverage $(\mathrm{R}=0.358$; $p=0.067$ ). Nevertheless, from 2012 to 2014, when incidence rates reached epidemic levels, such correlation was not observed $(\mathrm{R}=0.059 ; p=0.769)$.

\section{Discussion}

A global resurgence of pertussis has been observed beginning in the $1990 \mathrm{~s}$ and the early $21^{\text {st }}$ century. This study demonstrates a re-emergence of pertussis in Brazil, especially since 2012 . In the US, since 1993, the incidence of pertussis has increased, reaching over 25,000 cases per year in 2004 and 2005, 48,277 cases in 2012, and approximately 28,000 cases per year in 2013 and 2014 [15], with some localized outbreaks [2, 16, 17]. In Europe, most countries recorded an increase in the number of cases, especially the Netherlands, UK, Denmark and Norway. Between 2003 and 2007, the overall average annual incidence was $4.1 / 100,000$, rising to $4.9 / 100,000$ in 2009 [18-20]. In some countries, such as the Netherlands, abrupt increases occurred [21]. Data from this study suggest that epidemic levels were reached in Brazil in 2011, increased in 2012, and reached another peak in 2014. However, these values are still below those observed in other countries, such as in Europe, possibly because of underreporting.

In any epidemiological surveillance system, there is concern about underreporting. It has been demonstrated through capture-recapture methods that pertussis is significantly underreported; in the US, it is likely that the actual number of cases is 5-6 times than the reported 
Table 1 Frequency of different complications in fatal and non-fatal pertussis cases in different age groups in Brazil, 2007-2014. The rates displayed are the number of children with the specific complication/total number of children in each category (cured or fatal cases). Comparison of proportions was performed with the chi-square test

\begin{tabular}{|c|c|c|c|c|c|c|}
\hline \multirow[b]{2}{*}{ Age group } & & \multicolumn{5}{|l|}{ Complications } \\
\hline & & Pneumonia & Encephalopathy & Dehydration & Otitis & Malnutrition \\
\hline \multirow[t]{3}{*}{$0-2$ months } & Cured cases & 1237/6787 (18.2 \%) & 88/6763 (1.3 \%) & 159/6778 (2.3 \%) & 44/6748 (0.7 \%) & 71/6765 (1%) \\
\hline & Fatal cases & 233/370 (63 \%) & 24/346 (6.9 \%) & $34 / 351(9.7 \%)$ & $2 / 341(0.6 \%)$ & 16/349 (4.6 \%) \\
\hline & $p$-Value & $<0.001$ & $<0.001$ & $<0.001$ & 0.84 & $<0.001$ \\
\hline \multirow[t]{3}{*}{ 3-6 months } & Cured cases & $701 / 4550$ (15.4\%) & 49/4534 (1.1\%) & $117 / 4533$ (2.6 \%) & $64 / 4523(1.4 \%)$ & $50 / 4526(1.1 \%)$ \\
\hline & Fatal cases & 57/83 (68.7 \%) & 9/78 (11.5 \%) & 10/79 (12.6 \%) & 2/78 (2.6 \%) & 4/78 (5.1\%) \\
\hline & $p$-Value & $<0.001$ & $<0.001$ & $<0.001$ & 0.39 & 0.006 \\
\hline \multirow[t]{3}{*}{ 7-15 months } & Cured cases & 243/1764 (13.8 \%) & 14/1757 (0.8 \%) & 44/1758 (2.5 \%) & 46/1756 (2.6 \%) & 29/1758 (1.6\%) \\
\hline & Fatal cases & 13/21 (61.9 \%) & $1 / 19$ (5.2 \%) & 0 & $1 / 20(5 \%)$ & 0 \\
\hline & $p$-Value & $<0.001$ & 0.39 & - & 0.96 & - \\
\hline \multirow[t]{3}{*}{16 months-4 years } & Cured cases & 236/2612 (9 \%) & 15/2610 (0.6 \%) & $53 / 2615$ (2 \%) & $51 / 2607$ (2 \%) & 33/2609 (1.3\%) \\
\hline & Fatal cases & 3/10 (30 \%) & 0 & 0 & 0 & $2 / 10(20 \%)$ \\
\hline & $p$-Value & 0.08 & - & - & - & $<0.001$ \\
\hline \multirow[t]{3}{*}{ 5-14 years } & Cured cases & 115/2557 (4.5 \%) & 9/2550 (0.3\%) & $42 / 2558(1.6 \%)$ & $64 / 2555(2.5 \%)$ & 23/2551 (0.9\%) \\
\hline & Fatal cases & $1 / 4$ (25 \%) & $1 / 4(25 \%)$ & 0 & 0 & 0 \\
\hline & $p$-Value & 0.44 & $<0.001$ & - & - & - \\
\hline \multirow[t]{3}{*}{$>15$ years } & Cured cases & 83/2152 (3.9 \%) & 5/2151 (0.2 \%) & 18/2154 (0.8 \%) & $38 / 2153(1.8 \%)$ & 8/2152 (0.4 \%) \\
\hline & Fatal cases & $1 / 4(25 \%)$ & 0 & 0 & 0 & $1 / 4$ (25 \%) \\
\hline & $p$-Value & 0.37 & - & - & - & $<0.001$ \\
\hline
\end{tabular}

number. This failure to report cases may be even greater for teenagers and adults because the symptoms are usually mild and may go unnoticed [22-24]. Thus, the actual state of the disease in Brazil may be even more alarming.

Cyclic epidemic peaks of pertussis are observed in many countries. The interval between these peaks varies from one study to another, suggesting that they occur every 2-5 years $[2,6,18,25,26]$. Brazilian data show increases in the years 1997, 1998, 2004 and 2005. This study shows increasing incidence from 2011 to 2014; in 2014, it reached the highest number of cases in 30 years [27].
This study shows that pertussis has a clear seasonal pattern in Brazil, with an increase in the number of cases between late spring and summer (in the Southern Hemisphere), specifically in the months of November, December, January and February and especially in South and Southeast regions. Similarly, it has been reported that in the Northern Hemisphere, pertussis occurs most frequently in summer and autumn [18]. Interestingly, in Brazil, other acute respiratory tract infections are more common between the autumn and spring and peak in the winter [28]. These differences in seasonal patterns may be useful for identifying and diagnosing pertussis.

Table 2 Pertussis confirmation rates by vaccination status in different periods and age groups, Brazil, 2007-2014

\begin{tabular}{|c|c|c|c|c|}
\hline & \multirow[b]{2}{*}{ Notified cases (N) } & \multicolumn{3}{|l|}{ Confirmation rates } \\
\hline & & Fully vaccinated & Incompletely vaccinated or unvaccinated & $p$-value \\
\hline \multicolumn{5}{|l|}{ Period/Age group } \\
\hline \multicolumn{5}{|c|}{ Jan 2007 - May 2012} \\
\hline 7-15 months & 1797 & $379 / 1292(29,3 \%)$ & 212/505 (42\%) & $<0.001$ \\
\hline 16-48 months & 3319 & $809 / 2964(27.3 \%)$ & $132 / 355(37.2 \%)$ & $<0.001$ \\
\hline \multicolumn{5}{|l|}{ Period /Age group } \\
\hline \multicolumn{5}{|c|}{ Jun 2012-Dec 2014} \\
\hline 7-15 months & 3635 & $719 / 2722(26.4 \%)$ & 303/913 (33.2 \%) & $<0.001$ \\
\hline 16-48 months & 9283 & 2659/8548 (31.1\%) & 261/735 (35.5 \%) & 0.013 \\
\hline
\end{tabular}



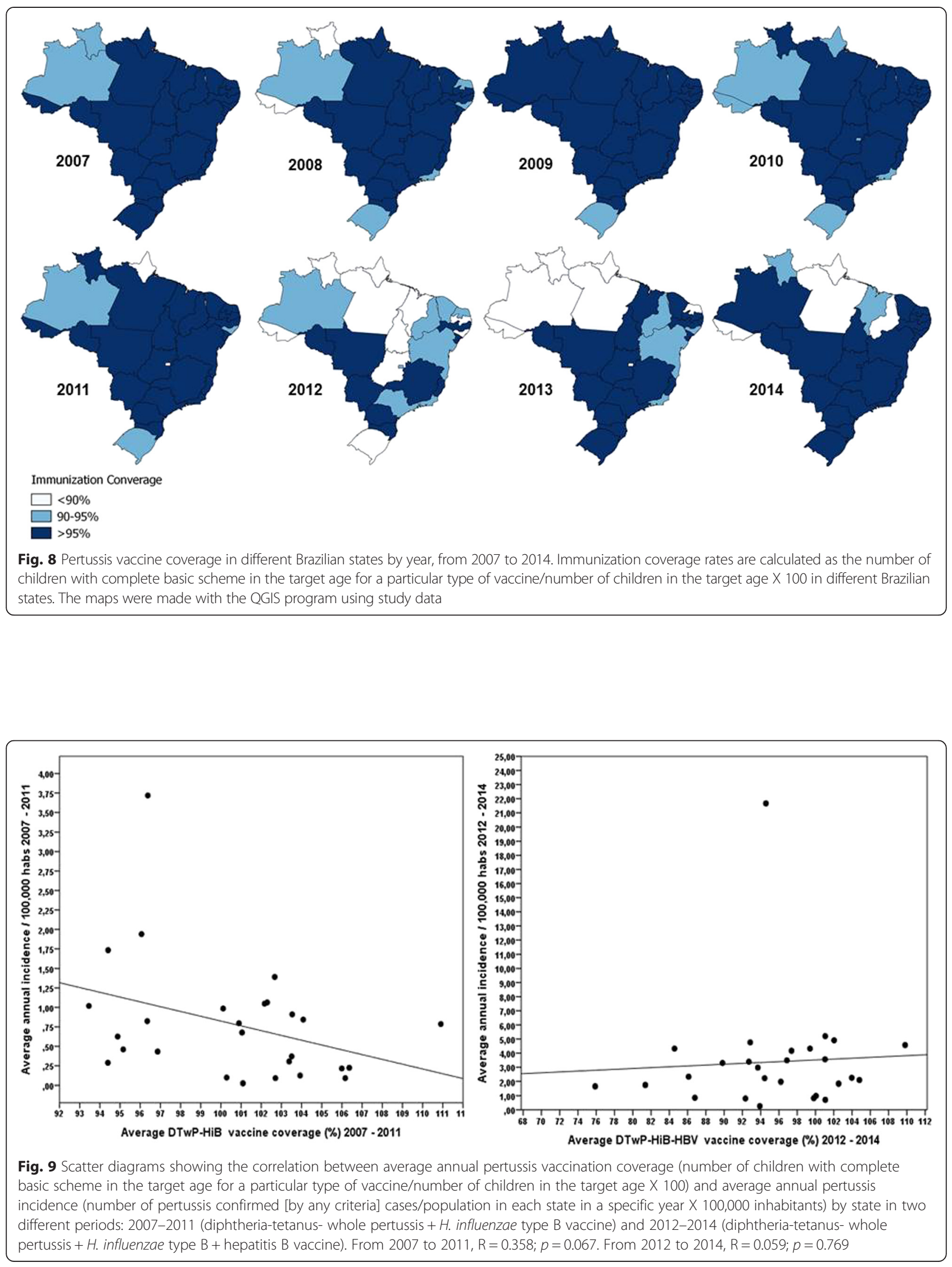
The age distribution of pertussis cases vary between the distinct regions of the world and from one period to another. During an epidemic in the US, the incidence was higher among children under 6 months of age; during the second epidemic, the incidence increased considerably for children aged 14-16 years [2, 17]. Similar variations in age distribution have been reported in Europe [20, 29].

In the present study, we found that $1 / 3$ of the confirmed cases occurred in infants aged 0 to 2 months, an age at which they have not received any dose of a pertussis vaccine. A little over half the cases occurred in children under the age of seven months, i.e., while they were in the process of immunization. During the period of increased incidence (2012-2014), the proportion of confirmed cases in not fully immunized subjects decreased, while an increase in the proportion of cases in older children and adolescents was observed.

Regarding the diagnostic criteria used, most cases were confirmed by clinical and epidemiological criteria, and laboratory confirmation was obtained in a little more than one-third of cases. This is a low proportion; however, it can be explained by the difficulties of obtaining a nasopharyngeal swab in most hospitals and health centres. Nevertheless, laboratory tests are important for confirming pertussis because clinical diagnosis can be difficult [30-32]. In the US, the significant increase in the number of cases may be associated with the introduction of more accurate techniques, such as PCR [16, 33]. In Mexico, there was a substantial increase in pertussis confirmations when PCR testing was implemented [26]. Interestingly, in Italy, the case definition is based on clinical criteria, and laboratory confirmation is not routine [29]. In Spain, the proportion of laboratory confirmation has gradually increased, reaching $69 \%$ [34]. In the US, each confirmed case is linked to a positive laboratory test or is epidemiologically associated with a case confirmed by culture or PCR [35]. Unfortunately, the culture of nasopharyngeal secretions of $B$. pertussis, despite high specificity, presents low sensitivity, and its applicability is subject to logistic limitations. The state of São Paulo, which has used the PCR technique since 2009, had the highest rate of laboratory confirmation of the country. In general, the Southern and Southeastern regions had the highest laboratory confirmation rates in Brazil. Efforts to improve pertussis laboratory diagnosis capacity in Brazil should be undertaken, health professionals involved in patient care in health services should be engaged, and laboratory infrastructure within the Unified Health System should be improved.

The pertussis complications are more prevalent in children under one year of age. In this study, pneumonia was the most commonly observed complication, especially in infants under 3 months of age. In California in 2010, $18.8 \%$ of patients with pertussis had pneumonia.
In Russia, the most frequent complications were encephalopathy, followed by bronchitis and pneumonia [17, 36]. A study in the state of Paraná in Brazil also showed that pneumonia was the complication most often associated with death [13]. The overall pertussis case fatality rate in Brazil is higher than that of other countries. In Canada between 1991 and 1997, there was a fatality rate of $0.9 \%$, less than half the rate observed in Brazil between 2007 and 2014 [37, 38]. Additionally, the absolute number of deaths from pertussis in Brazil is higher than those observed in other countries. In England between 2001 and 2011, there were 48 deaths, the same number of deaths reported in a 6-month period during the epidemic years of the disease in Brazil [39]. In the US in 2013, there were only 13 deaths from pertussis, with a case fatality rate of $0.02 \%$ [40].

A global resurgence of pertussis has been noticed, especially in developed countries. Major hypotheses to explain it include a lower immunogenicity of aP compared with $\mathrm{wP}$ vaccines, which would lead to the early weaning of vaccine-induced immunity, the circulation of $B$. pertussis among adolescents and adults and, from these groups, the infection of infants who are not fully immunized. This phenomenon may also be associated with vaccines' selective pressure on circulating strains, which could cause them to block the antibodies produced by immunization [39].

However, in Brazil, aP vaccines have never been introduced universally and are available only in private vaccination clinics. The proportion of Brazilian children and adults who have been immunized with aP vaccines is not known. Vaccines containing the aP component are available in the NIP only to pregnant women and children who have developed serious side effects after the use of $\mathrm{wP}$ vaccines. Thus, the increase in the incidence of pertussis observed in recent years in Brazil should have other possible explanations.

This study reveals that most cases of pertussis reported in Brazil from 2007 to 2014 occurred in subjects older than 6 months of age, with almost $13 \%$ of these cases recorded in fully immunized infants and children aged 7 months to 4 years. Almost $1 / 3$ of these cases were laboratory confirmed. These data point to the possibility of some degree of vaccine escape in the context of the DTwP-Hib-HBV vaccine. We observed that among the reported cases, the proportion of confirmed cases was significantly lower among fully vaccinated children, demonstrating the protective effect of the vaccines, although our study did not aim to evaluate the effectiveness of the vaccines currently being used in Brazil. Nevertheless, the difference in vaccination rates among the confirmed and unconfirmed cases was lower during the period of June 2012 through December 2014 compared with the period of January 2007 through May 2012. 
This may point to a lower performance of DTwP-HibHBV compared with the DTwP-Hib.

Interestingly, the increase in the incidence of pertussis in Brazil coincides with the introduction of the DTwPHib-HBV to the NIP in 2012, although an initial increase in the incidence was already evident in 2011, before the DTwP-Hib was replaced. It is also worth noting that during this period, there was a relative reduction in vaccine coverage, which increased the number of states that did not reach $95 \%$ coverage. In the present study, we found that $2 \%$ of the confirmed cases occurred in incompletely vaccinated infants older than 6 months of age. During the pre-epidemic period there was a trend for states with lower vaccination coverage present the highest rates of incidence of pertussis. This pattern was not observed in the 2012-2014 period.

In 2013, Brazil introduced the DaPT for pregnant women as a measure to contain the resurgence of pertussis. Brazil's main challenges in facing pertussis resurgence will be to offer the highest quality medical attention to reduce mortality, to improve the infrastructure for laboratory diagnoses, and to maintain high vaccination coverage. Additional studies to assess the effectiveness of the current vaccination schedule, including basic research on the genetics and evolution of circulating $B$. pertussis strains, are also needed.

\section{Conclusions}

There has been a significant increase in the incidence of pertussis in Brazil, especially since 2012. A little over half of the cases occurred in infants younger than 7 months of age. Some of the confirmed cases may be associated with primary vaccine failure, as some have occurred in vaccinated children aged 7 months to 4 years. Laboratory confirmation rates are less than ideal and have large regional differences. Fatality rates are high compared with those described in the literature; they are significantly higher in infants and are strongly associated with such complications as pneumonia, malnutrition and dehydration. The increase in incidence coincides with the introduction of the pentavalent vaccine and with a relative reduction in vaccination coverage in several states. Brazil's challenges in facing rising pertussis rates will be to offer the highest quality medical care, improve the infrastructure for laboratory diagnostics and maintain high vaccination coverage.

\section{Consent}

Consent was not considered necessary by the Research Ethics Committee of the Instituto Oswaldo Cruz,as it is a study with secondary data from reporting and surveillance records.

\section{Abbreviations}

DTP: Diphtheria-tetanus-pertussis vaccine; WP: whole pertussis vaccine; aP: acelular pertussis vaccine; NIP: National Immunization Program;
DTwP: Diphtheria-tetanus- whole pertussis vaccine; DTwP-Hib: DTwP $+H$. influenzae type B vaccine; DTwP-HiB-HBV: DTwP + H. influenzae type B + hepatitis B; DTaP: Diphtheria-tetanus- acelular pertussis vaccine; DataSUS: Department of Informatics of the Unified Health System SINAN: Information System for Notifiable Diseases; PCR: Polymerase chain reaction; LACEN: Central Laboratory of Public Health; QGIS: Quantum Geographic Information System.

\section{Competing interests}

The authors declare that they have no competing interests.

\section{Authors' contributions}

LMG and ELNCC acquired, analyzed and interpreted data, FACC conceived the study design, supervised data analyses and wrote the manuscript. All authors read and approved the final version of the manuscript.

\section{Acknowledgements}

We thank the Ministry of Health of Brazil for providing secondary data used in this study. The study was conducted with funding from Fiocruz and the Brazilian Federal Agency for Support and Evaluation of Graduate Education (CAPES). FACC is recipient of a research productivity grant level 2 of the Brazilian National Council for Scientific and Technological Development (CNPq).

\section{Author details}

${ }^{1}$ Regional Office Oswaldo Cruz Foundation (Fiocruz), Teresina, Piauí, Brazil. 'Laboratory of Epidemiology and Molecular Systematics, Oswaldo Cruz Institute, Fiocruz, Rio de Janeiro, Brazil. ${ }^{3}$ Federal Institute of Education, Science and Technology of Piauí (IFPI), Teresina, Piauí, Brazil.

Received: 22 July 2015 Accepted: 15 October 2015

Published online: 23 October 2015

\section{References}

1. Berti E, Chiappini E, Orlandini E, Galli L, de Martino M. Pertussis is still common in a highly vaccinated infant population. Acta Paediatr. 2014:103(8):846-9.

2. Winter $K$, Glaser C, Watt J, Harriman $K$, (CDC) CfDCaP. Pertussis epidemicCalifornia, 2014. MMWR Morb Mortal Wkly Rep. 2014;63(48):1129-32.

3. Tizolova A, Brun D, Guiso N, Guillot S. Development of real-time PCR assay for differential detection of Bordetella bronchiseptica and Bordetella parapertussis. Diagn Microbiol Infect Dis. 2014;78(4):347-51.

4. Leber AL. Pertussis: relevant species and diagnostic update. Clin Lab Med. 2014;34(2):237-55

5. Ferrer A, Calicó I, Manresa JM, Andreu A, Moraga F, Valle I. Microorganisms isolated in cases of pertussis-like syndrome. Enferm Infecc Microbiol Clin. 2000;18(9):433-8

6. Mattoo S, Cherry JD. Molecular pathogenesis, epidemiology, and clinical manifestations of respiratory infections due to Bordetella pertussis and other Bordetella subspecies. Clin Microbiol Rev. 2005;18(2):326-82.

7. Jakinovich A, Sood SK. Pertussis: still a cause of death, seven decades into vaccination. Curr Opin Pediatr. 2014;26(5):597-604.

8. Chiappini E, Stival A, Galli L, de Martino M. Pertussis re-emergence in the post-vaccination era. BMC Infect Dis. 2013;13:151

9. Galindo BM, Concepción D, Galindo MA, Pérez A, Saiz J. Vaccine-related adverse events in Cuban children, 1999-2008. MEDICC Rev. 2012;14(1):38-43.

10. Lambert LC. Pertussis vaccine trials in the 1990s. J Infect Dis. 2014:209 Suppl 1:S4-9.

11. Klein NP. Licensed pertussis vaccines in the United States. History and current state. Hum Vaccin Immunother. 2014;10(9):2684-90.

12. Zhang L, Prietsch SO, Axelsson I, Halperin SA. Acellular vaccines for preventing whooping cough in children. Cochrane Database Syst Rev. 2014;9:CD001478.

13. Torres RS, Santos TZ, Torres RA, Pereira W, Fávero LA, Filho OR, et al. Resurgence of pertussis at the age of vaccination: clinical, epidemiological, and molecular aspects. J Pediatr (Rio J). 2015:91(4):333-8.

14. Departamento de Informações do Sistema Único de Saúde do Brasil DATASUS [http://pni.datasus.gov.br/index.asp] Accessed 21 March 2015.

15. Centers for Disease Control and Prevention. Pertussis (whopping cough) Pertussis cases by year (1922 - 2014). Available in:http://www.cdc.gov/ pertussis/surv-reporting/cases-by-year.html. Accessed March 20th2015. 
16. (CDC) CfDCaP. Pertussis epidemic-Washington, 2012. MMWR Morb Mortal Wkly Rep. 2012;61(28):517-22

17. Winter K, Harriman K, Zipprich J, Schechter R, Talarico J, Watt J, et al. California pertussis epidemic, 2010. J Pediatr. 2012;161(6):1091-6.

18. European Centre for Disease Prevention and Control. Annual epidemiological report 2014 - Vaccinepreventablediseases - invasive bacterial diseases. Stockholm: ECDC; 2015.

19. EUVAC.NET. Pertussis surveillance report 2003 - 2007. Available in: http:// ecdc.europa.eu/en/publications/Publications/ pertussis_report_20032007_euvacnet.pdf. Accessed 19 March 2015.

20. EUVAC.NET. Pertussis surveillance report 2009. Available in: http:// www.euvac.net/graphics/euvac/pdf/pertussis_2009.pdf. Accessed 19 March 2015.

21. de Melker HE, Conyn-van Spaendonck MA, Rümke HC, van Wijngaarden JK Mooi FR, Schellekens JF. Pertussis in The Netherlands: an outbreak despite high levels of immunization with whole-cell vaccine. Emerg Infect Dis. 1997:3(2):175-8

22. Edwards KM. Overview of pertussis: focus on epidemiology, sources of infection, and long term protection after infant vaccination. Pediatr Infect Dis J. 2005;24(6 Suppl):S104-8.

23. Shaikh R, Guris D, Strebel PM, Wharton M. Underreporting of pertussis deaths in the United States: need for improved surveillance. Pediatrics. 1998;101(2):323.

24. Sutter RW, Cochi SL. Pertussis hospitalizations and mortality in the United States, 1985-1988. Evaluation of the completeness of national reporting. JAMA. 1992;267(3):386-91.

25. Quinn HE, McIntyre PB. Pertussis epidemiology in Australia over the decade 1995-2005-trends by region and age group. Commun Dis Intell Q Rep. 2007:31(2):205-15.

26. Pérez-Pérez GF, Rojas-Mendoza T, Cabrera-Gaytán DA, Grajales-Muñizd C. Panorama epidemiológico de la tos ferina:19 años de estudio epidemiológico en el Instituto Mexicano del Seguro Social. Rev Med Inst Mex Seguro Soc. 2015;53(2):164-70.

27. Sistema de Informação de Agravos de Notificação - SINAN [http:// dtr2004.saude.gov.br/sinanweb/tabnet/dh?sinannet/coqueluche/bases/ coquebrnet.def] 21 March 2015.

28. Salomão Junior JB, Gardinassi LG, Simas PV, Bittar CO, Souza FP, Rahal P, et al. Human respiratory syncytial virus in children hospitalized for acute lower respiratory infection. J Pediatr (Rio J). 2011;87(3):219-24.

29. Gabutti G, Rota MC. Pertussis: a review of disease epidemiology worldwide and in Italy. Int J Environ Res Public Health. 2012;9(12):4626-38.

30. de Carvalho AP, Pereira EM. Acellular pertussis vaccine for adolescents. J Pediatr (Rio J). 2006;82(3 Suppl):S15-24.

31. Schellekens J, von König CH, Gardner P. Pertussis sources of infection and routes of transmission in the vaccination era. Pediatr Infect Dis J. 2005;24(5 Suppl):S19-24.

32. Hay JW, Ward JI. Economic considerations for pertussis booster vaccination in adolescents. Pediatr Infect Dis J. 2005;24(6 Suppl):S127-33.

33. Spokes PJ, Quinn HE, McAnulty JM. Review of the 2008-2009 pertussis epidemic in NSW: notifications and hospitalisations. N S W Public Health Bull. 2010;21(7-8):167-73.

34. Pardo-Serrano FJ. Situation of pertussis in the community of Valencia: an epidemic revival? Enferm Infecc Microbiol Clin. 2014;32(4):270-1.

35. Faulkner A, Skoff T, Martin S, Cassiday P, Tondella ML, Liang J, Ejigiri OG: Manual for surveillance of vaccine-preventable diseases. Chapter 10: Pertussis. Available in: http://www.cdc.gov/vaccines/pubs/survmanual/ chpt10-pertussis.html. Accessed 19 March 2015.

36. Borisova O, Kombarova SY, Zakharova NS, van Gent M, Aleshkin VA, Mazurova I, et al. Antigenic divergence between Bordetella pertussis clinical isolates from Moscow, Russia, and vaccine strains. Clin Vaccine Immunol. 2007; 14(3):234-8.

37. Halperin SA, Wang EE, Law B, Mills E, Morris R, Déry P, et al. Epidemiological features of pertussis in hospitalized patients in Canada, 1991-1997: report of the Immunization Monitoring Program-Active (IMPACT). Clin Infect Dis. 1999;28(6):1238-43.

38. Halperin SA, Bortolussi R, Langley JM, Eastwood BJ, De Serres G. A randomized, placebo-controlled trial of erythromycin estolate chemoprophylaxis for household contacts of children with culture-positive bordetella pertussis infection. Pediatrics. 1999;104(4):e42.

39. van Hoek AJ, Campbell H, Amirthalingam G, Andrews N, Miller E. The number of deaths among infants under one year of age in England with pertussis: results of a capture/recapture analysis for the period 2001 to 2011. Euro Surveill. 2013;18(9). Available in: http://www.eurosurveillance.org/ ViewArticle.aspx?Articleld=20414. Accessed 23 March 2015.

40. Centers for Disease Control and Prevention. 2013 Final Pertussis Surveillance Report. Available in: http://www.cdc.gov/pertussis/downloads/pertuss-survreport-2013.pdf. Accessed 19 March 2015.

\section{Submit your next manuscript to BioMed Central and take full advantage of:}

- Convenient online submission

- Thorough peer review

- No space constraints or color figure charges

- Immediate publication on acceptance

- Inclusion in PubMed, CAS, Scopus and Google Scholar

- Research which is freely available for redistribution 